\title{
Nonstandard Finite Difference Variational Integrators for Multisymplectic PDEs
}

\author{
Cuicui Liao and Xiaohua Ding \\ Department of Mathematics, Harbin Institute of Technology, 2 Wenhua West Road, Shandong, \\ Weihai 264209, China \\ Correspondence should be addressed to Xiaohua Ding, mathdxh@hit.edu.cn
}

Received 12 August 2012; Accepted 4 October 2012

Academic Editor: Renat Zhdanov

Copyright (C) 2012 C. Liao and X. Ding. This is an open access article distributed under the Creative Commons Attribution License, which permits unrestricted use, distribution, and reproduction in any medium, provided the original work is properly cited.

\begin{abstract}
We use the idea of nonstandard finite difference methods to derive the discrete variational integrators for multisymplectic PDEs. We obtain a nonstandard finite difference variational integrator for linear wave equation with a triangle discretization and two nonstandard finite difference variational integrators for the nonlinear Klein-Gordon equation with a triangle discretization and a square discretization, respectively. These methods are naturally multisymplectic. Their discrete multisymplectic structures are presented by the multisymplectic form formulas. The convergence of the discretization schemes is discussed. The effectiveness and efficiency of the proposed methods are verified by the numerical experiments.
\end{abstract}

\section{Introduction}

It is a fundamental approach to develop the discrete multisymplectic numerical methods based on the discrete Hamilton's principle, because it leads in a natural way to multisymplectic integrators [1]. The discrete Euler-Lagrange equation is produced in the discrete variational principle [2-4]; meanwhile, the discrete multisymplectic structure is also generated $[5,6]$. In the other words, the discrete variational integrators are multisymplectic automatically.

\subsection{Multisymplectic Structure of Discrete Variational Integrators}

By the Hamilton's principle [6-9], the discrete multisymplectic structure which is preserved by the discrete variational integrator, is described by Poincare-Cartan forms, in a differential geometric language. In paper [6], Marsden et al. showed how to obtain this structure directly 
from the variational principle, on the Lagrangian side. They defined it as the multisymplectic form formula, and they showed that it was conserved by the discrete variational integrator.

Lemma 1.1. If $u$ is a solution of discrete Euler-Lagrange equation and $V, W$ are first variations of $u$, then the following discrete multsisymplectic form formula holds:

$$
\sum_{\Delta ; \Delta \cap \partial U \neq 0}\left(\sum_{l: \Delta^{l} \in \partial U}\left[\left(j^{1} u\right) *\left(i_{j^{1} V} i_{j^{1} W} \Omega_{L}^{l}\right)\right](\Delta)\right)=0 .
$$

The details of this conclusion could be referred to papers [5,6]. This conclusion states that the discrete variational principles produce discrete variational integrators, and the multisymplecticity of these variational integrators is presented by the discrete multisymplectic form formula (1.1).

Vankerschaver et al. [10] revisited the multisymplectic form formula [6], showing that it could be obtained from the boundary Lagrangian that they defined in their paper. They presented an easy way to derive discrete multisymplectic form formula from discrete variational principle, using the notations of Poincaré-Cartan forms. In this paper, we follow the same way to derive the discrete multisymplectic form formulas of our discrete variational integrators.

When we use the discrete variational principle, we need to make a approximation of the Lagrangian. Here, in our paper, we would use nonstandard finite difference methods, instead of standard finite difference, to approximate the Lagrangian function, and derive the corresponding discrete variational integrators.

\subsection{Nonstandard Finite Difference Methods}

The nonstandard finite difference schemes are well developed by Mickens [11-15] in the past decades. These schemes are developed for compensating the weaknesses that may be caused by standard finite difference methods, for example, the numerical instabilities. Regarding the positivity of solutions, boundedness, and monotonicity of solutions, nonstandard finite difference schemes have a better performance than standard finite difference schemes, due to its flexibility to construct a nonstandard finite difference scheme that can preserve certain properties and structures, which are obeyed by the original equations. Also, the dynamic consistency could be presented well by nonstandard finite difference scheme. These advantages of nonstandard finite difference methods have been shown in many numerical applications. GonzLez-Parra et al. [16-18] developed nonstandard finite difference methods to solve population or biological models. The positivity condition and the conservation law of population dynamics are preserved by nonstandard finite difference schemes. Jordan [19] and Malek [20] constructed nonstandard finite difference schemes for heat transfer problems. For the symplectic systems, Mickens [15] derived the nonstandard finite difference variational integrator for symplectic ODEs. Ma et al. [21] developed the nonstandard finite difference variational integrator in stochastic ordinary differential equations.

The initial foundation of nonstandard finite difference methods is formed by the exact finite difference schemes [22]. After generalizing these results, Mickens summarizes the following three basic rules to construct nonstandard finite difference schemes. 
(1) The orders of the discrete derivatives should be equal to the orders of the corresponding derivatives appearing in the differential equations.

Note. If the orders of the discrete derivatives are larger than those occurring in the differential equations, then numerical instabilities will in general occur.

(2) Discrete representations for derivatives must, in general, have nontrivial denominator functions.

Note. For example, the discrete first-derivative is generally represented by

$$
\frac{d u}{d t} \longrightarrow \frac{u_{i+1}-\varphi(\Delta t) u_{i}}{\phi(\Delta t)}
$$

where the numerator functions $\psi(\Delta t)$ and the denominator functions $\phi(\Delta t)$ satisfy

$$
\varphi(\Delta t)=1+\mathcal{O}((\Delta t)), \quad \phi(\Delta t)=\Delta t+\mathcal{O}\left((\Delta t)^{2}\right) .
$$

(3) Both linear and nonlinear terms should be represented by nonlocal discrete representations on the discrete computational lattice.

Note. For example,

$$
\begin{gathered}
u \longrightarrow 2 u_{i}-u_{i+1}, \\
u^{2} \longrightarrow u_{i} u_{i+1}, \\
u^{2} \longrightarrow\left(\frac{u_{i-1}+u_{i}+u_{i+1}}{3}\right) u_{i}, \\
u^{3} \longrightarrow 2 u_{i}^{3}-u_{i}^{2} u_{i+1}, \\
u^{3} \longrightarrow u_{i-1} u_{i} u_{i+1} .
\end{gathered}
$$

In our paper, we combine the advantages of nonstandard finite difference methods and discrete variational principles to construct nonstandard finite difference variational integrators, for two multisymplectic PDEs. These integrators are multysimplectic and their multysimplecticity are presented by their discrete multisymplectic form formulas, respectively.

In Section 2, we consider a simple linear wave equation. With the triangle discretization, we define the discrete Lagrangian using the idea of nonstandard finite difference and derive discrete variational integrator and the corresponding multisymplectic form formula, by discrete variational principle. The convergence of this method is analyzed. In Section 3, for the nonlinear Klein-Gordon equation, triangle discretization and square discretization are considered to obtain the nonstandard finite difference variational integrators. The discrete multisymplectic structures are presented, respectively. The convergence orders of the two methods are also discussed, and the convergence orders are shown in error tables in the numerical experiment section. Section 4 is devoted to showing the numerical behaviors of the developed nonstandard finite difference variational integrators. 


\section{Nonstandard Finite Difference Variational Integrator for Linear Wave Equation}

We first consider a simple linear wave equation,

$$
u_{t t}=u_{x x}
$$

where $u(x, t)$ is a scalar field function with two independent variables, $x$ and $t$.

This linear wave equation is actually a multisymplectic PDE. As a classical and simple multisymplectic example, wave equation and its multisymplectic structure have been studied from both Hamiltonian [23-28] and Lagrangian viewpoints [3, 5, 6, 10]. Based on the Lagrangian viewpoint, we could also obtain (2.1) from the Euler-Lagrange equation

$$
\frac{\partial L}{\partial u}=\frac{d}{d t} \frac{\partial L}{\partial u_{t}}+\frac{d}{d x} \frac{\partial L}{\partial u_{x}}
$$

with the Lagrangian function $L\left(u, u_{t}, u_{x}\right)$,

$$
L\left(u, u_{t}, u_{x}\right)=\frac{1}{2} u_{t}^{2}-\frac{1}{2} u_{x}^{2}
$$

Assume that we have a uniform quadrangular mesh in the base space, with mesh lengths $\Delta x$ and $\Delta t$. The nodes in this mesh are denoted by $(i, j) \in \mathbb{Z} \times \mathbb{Z}$, corresponding to the points $\left(x_{i}, t_{j}\right):=(i \Delta x, j \Delta t)$ in $\mathbb{R}^{2}$. We denote the value of the field $u$ at the node $(i, j)$ by $u_{i}^{j}$. We label the triangle at $(i, j)$ with three ordered triple $((i, j),(i+1, j),(i, j+1))$ as $\triangle_{i j}$, and we define $X_{\Delta}$ to be the set of all such triangles. Then the discrete jet bundle $[6,10]$ is defined as follows:

$$
J_{\Delta}^{1} Y:=\left\{\left(u_{i}^{j}, u_{i+1}^{j}, u_{i}^{j+1}\right) \in \mathbb{R}^{3}:((i, j),(i+1, j),(i, j+1)) \in X_{\Delta}\right\}
$$

which is equal to $X_{\Delta} \times \mathbb{R}^{3}$.

Now we use nonstandard finite difference to define the discrete Lagrangian $L_{d}$ on $J_{\Delta}^{1} Y$, which is the discrete version of Lagrangian density [10, 29],

$$
L_{d}\left(u_{i}^{j}, u_{i+1}^{j}, u_{i}^{j+1}\right):=\frac{1}{2} \Delta t \Delta x L\left(\frac{u_{i}^{j}+u_{i+1}^{j}+u_{i}^{j+1}}{3}, \frac{u_{i}^{j+1}-u_{i}^{j}}{\phi(\Delta t)}, \frac{u_{i+1}^{j}-u_{i}^{j}}{\psi(\Delta x)}\right),
$$

where denominator functions $\phi(\Delta t)$ and $\psi(\Delta x)$ are defined according the exact solution of wave equation $[5,6,12]$

$$
\phi(\Delta t)=\frac{1}{2} \sin \left(\frac{\Delta t}{2}\right), \quad \psi(\Delta x)=\frac{1}{2} \sin \left(\frac{\Delta x}{2}\right) .
$$

We have followed the rules of constructing nonstandard finite difference schemes in Mickens' papers [11-15]. 
Journal of Applied Mathematics

(1) The discrete first-derivative is represented by

$$
\frac{d u}{d t} \longrightarrow \frac{u_{i}^{j+1}-u_{i}^{j}}{\phi(\Delta t)}, \quad \frac{d u}{d x} \longrightarrow \frac{u_{i+1}^{j}-u_{i}^{j}}{\psi(\Delta x)}
$$

where denominator functions $\phi(\Delta t), \psi(\Delta x)$ are defined in (2.6). Using Taylor series expansion,

$$
\sin \left(\frac{\Delta t}{2}\right)=\frac{\Delta t}{2}-\frac{1}{48}(\Delta t)^{3}+\cdots
$$

Then the denominator functions satisfy

$$
\phi(\Delta t)=\Delta t+\mathcal{O}\left((\Delta t)^{3}\right), \quad \psi(\Delta x)=\Delta x+\mathcal{O}\left((\Delta x)^{3}\right) .
$$

(2) Nonlocal representation on the discrete computational lattice are used here by

$$
u \rightarrow \frac{u_{i}^{j}+u_{i+1}^{j}+u_{i}^{j+1}}{3}
$$

So, for the linear wave equation (2.1) with the Lagrangian (2.3), the discrete Lagrangian becomes

$$
L_{d}\left(u_{i}^{j}, u_{i+1}^{j}, u_{i}^{j+1}\right)=\frac{1}{2} \Delta t \Delta x\left(\frac{1}{2}\left(\frac{u_{i}^{j+1}-u_{i}^{j}}{\phi(\Delta t)}\right)^{2}-\frac{1}{2}\left(\frac{u_{i+1}^{j}-u_{i}^{j}}{\psi(\Delta x)}\right)^{2}\right) .
$$

By the discrete Hamilton's principle $[6,10]$, we have the discrete Euler-Lagrange equation,

$$
D_{1} L_{d}\left(u_{i}^{j}, u_{i+1}^{j}, u_{i}^{j+1}\right)+D_{2} L_{d}\left(u_{i-1}^{j}, u_{i}^{j}, u_{i-1}^{j+1}\right)+D_{3} L_{d}\left(u_{i}^{j-1}, u_{i+1}^{j-1}, u_{i}^{j}\right)=0
$$

where $L_{d}\left(u_{i-1}^{j}, u_{i}^{j}, u_{i-1}^{j+1}\right)$ and $L_{d}\left(u_{i}^{j-1}, u_{i+1}^{j-1}, u_{i}^{j}\right)$ are defined similarly as (2.11), which are

$$
\begin{aligned}
& L_{d}\left(u_{i-1}^{j}, u_{i}^{j}, u_{i-1}^{j+1}\right)=\frac{1}{2} \Delta t \Delta x\left(\frac{1}{2}\left(\frac{u_{i-1}^{j+1}-u_{i-1}^{j}}{\phi(\Delta t)}\right)^{2}-\frac{1}{2}\left(\frac{u_{i}^{j}-u_{i-1}^{j}}{\psi(\Delta x)}\right)^{2}\right), \\
& L_{d}\left(u_{i}^{j-1}, u_{i+1}^{j-1}, u_{i}^{j}\right)=\frac{1}{2} \Delta t \Delta x\left(\frac{1}{2}\left(\frac{u_{i}^{j}-u_{i}^{j-1}}{\phi(\Delta t)}\right)^{2}-\frac{1}{2}\left(\frac{u_{i+1}^{j-1}-u_{i}^{j-1}}{\psi(\Delta x)}\right)^{2}\right) .
\end{aligned}
$$


After some simple calculations, the discrete Euler-Lagrange equation (2.12) becomes

$$
\frac{u_{i}^{j+1}-2 u_{i}^{j}+u_{i}^{j-1}}{(\phi(\Delta t))^{2}}-\frac{u_{i+1}^{j}-2 u_{i}^{j}+u_{i-1}^{j}}{(\psi(\Delta x))^{2}}=0 .
$$

We could find that this scheme is symmetric in $(i, j+1)$ and $(i, j-1),(i+1, j)$ and $(i-1, j)$. This is the nonstandard finite difference variational integrator, for the linear wave equation.

As we mentioned in Section 1 and Lemma 1.1, the advantages of deriving the multisymplectic numerical schemes from discrete variational principle are that they are naturally multisymplectic and the discrete multisymplectic structures are also generated in the variational principle. Now it is meaningful to show the multisymplectic structure of this discrete variational integrator (2.14) based on nonstandard finite difference method.

Since we consider triangulation discretization here, we focus on three adjacent triangles around $u_{i}^{j}$, denote this area by $U$. Following the idea in [10], the discrete boundary Lagrangian is given by

$$
L_{\partial U}\left(u_{\partial U}\right):=\operatorname{ext}_{u_{i}^{n}}\left[L_{d}\left(u_{i}^{n}, u_{i+1}^{n}, u_{i}^{n+1}\right)+L_{d}\left(u_{i-1}^{n}, u_{i}^{n}, u_{i-1}^{n+1}\right)+L_{d}\left(u_{i}^{n-1}, u_{i+1}^{n-1}, u_{i}^{n}\right)\right],
$$

where

$$
u_{\partial U}:=\left(u_{i+1}^{n}, u_{i}^{n+1}, u_{i-1}^{n+1}, u_{i-1}^{n}, u_{i}^{n-1}, u_{i+1}^{n-1}\right)
$$

Taking twice exterior derivative of both sides, we have, by the fact that $\mathbf{d}^{2} L_{\partial U} \equiv 0$, the discrete multisymplectic form formula with following form [10]:

$$
\sum_{k=1}^{3} \sum_{l=1 ; l \neq k}^{3} \Omega_{L}^{k}\left(\Delta^{(l)}\right)=0
$$

where $\Omega_{L}^{k}=-\mathbf{d} \Theta_{L}^{k}$ (for $\left.k=1,2,3\right)$. The discrete Poincaré-Cartan forms $\Theta_{L}^{1}, \Theta_{L}^{2}$, and $\Theta_{L}^{3}$ are defined by

$$
\Theta_{L}^{1}\left(u_{i}^{n}, u_{i+1}^{n}, u_{i}^{n+1}\right):=D_{1} L_{d}\left(u_{i}^{n}, u_{i+1}^{n}, u_{i}^{n+1}\right) d u_{i}^{n},
$$

and similarly for $\Theta_{L}^{2}$ and $\Theta_{L}^{3}$. Thus, for the linear wave equation (2.1), the multisymplectic form formula of this scheme (2.14) based on nonstandard finite difference method can be obtained as follows:

$$
\frac{d u_{i}^{j+1} \wedge d u_{i}^{j}+d u_{i}^{j-1} \wedge d u_{i}^{j}}{(\phi(\Delta t))^{2}}-\frac{d u_{i+1}^{j} \wedge d u_{i}^{j}+d u_{i-1}^{j} \wedge d u_{i}^{j}}{(\psi(\Delta x))^{2}}=0 .
$$

Now we have the first conclusion. 
Theorem 2.1. The nonstandard finite difference variational integrator (2.14),

$$
\frac{u_{i}^{j+1}-2 u_{i}^{j}+u_{i}^{j-1}}{(\phi(\Delta t))^{2}}-\frac{u_{i+1}^{j}-2 u_{i}^{j}+u_{i-1}^{j}}{(\psi(\Delta x))^{2}}=0
$$

for linear wave equation (2.1) is multisymplectic, and the discrete multisymplectic structure is

$$
\frac{d u_{i}^{j+1} \wedge d u_{i}^{j}+d u_{i}^{j-1} \wedge d u_{i}^{j}}{(\phi(\Delta t))^{2}}-\frac{d u_{i+1}^{j} \wedge d u_{i}^{j}+d u_{i-1}^{j} \wedge d u_{i}^{j}}{(\psi(\Delta x))^{2}}=0
$$

We now discuss the convergence of this variational integrator (2.14) based on the nonstandard finite difference method. From the Lax equivalence theorem we know that, for a well-posed linear initial value problem, the consistent finite difference method is convergent if and only if it is stable.

By Taylor series expansion, we have

$$
\begin{aligned}
\frac{u_{i}^{j+1}-2 u_{i}^{j}+u_{i}^{j-1}}{(\phi(\Delta t))^{2}} & =\frac{u_{i}^{j+1}-2 u_{i}^{j}+u_{i}^{j-1}}{\left(\Delta t+\mathcal{O}\left((\Delta t)^{3}\right)\right)^{2}}=\frac{1-\mathcal{O}\left((\Delta t)^{2}\right)}{(\Delta t)^{2}}\left(u_{i}^{j+1}-2 u_{i}^{j}+u_{i}^{j-1}\right) \\
& =\frac{1-\mathcal{O}\left((\Delta t)^{2}\right)}{(\Delta t)^{2}}\left((\Delta t)^{2} u_{t t}\left(x_{i}, t_{j}\right)+\mathcal{O}(\Delta t)^{4}\right) \\
& =u_{t t}\left(x_{i}, t_{j}\right)+\mathcal{O}\left((\Delta t)^{2}\right) .
\end{aligned}
$$

Similarly,

$$
\frac{u_{i+1}^{j}-2 u_{i}^{j}+u_{i-1}^{j}}{(\psi(\Delta x))^{2}}=u_{x x}\left(x_{i}, t_{j}\right)+\mathcal{O}\left((\Delta x)^{2}\right)
$$

The above two equations show that the scheme is consistent and the truncation error for the integrator $(2.14)$ is $\mathcal{O}\left((\Delta t)^{2}+(\Delta x)^{2}\right)$.

To explore the stability of the nonstandard finite difference variational integrator (2.14), we introduce the following notations:

$$
v_{i}^{j}=\frac{u_{i}^{j}-u_{i}^{j-1}}{\phi(\Delta t)}, \quad w_{i-1 / 2}^{j}=\frac{u_{i}^{j}-u_{i-1}^{j}}{\psi(\Delta t)} .
$$


Then the three-level explicit integrator (2.14) is equivalent to the following two-level scheme:

$$
\begin{gathered}
\frac{v_{i}^{j+1}-v_{i}^{j}}{\phi(\Delta t)}=\frac{w_{i+1 / 2}^{j}-w_{i-1 / 2}^{j}}{\psi(\Delta x)}, \\
\frac{w_{i-1 / 2}^{j+1}-w_{i-1 / 2}^{j}}{\phi(\Delta t)}=\frac{v_{i}^{j+1}-v_{i-1}^{j+1}}{\psi(\Delta x)} .
\end{gathered}
$$

By using the Von Neumann method [30], we could get the amplification matrix of the above scheme,

$$
G(\beta, \Delta t)=\left(\begin{array}{cc}
1 & 2 i r \sin (\beta \Delta x) \\
2 i r \sin (\beta \Delta x) & 1-4 r^{2} \sin ^{2}(\beta \Delta x)
\end{array}\right)
$$

where $r=\phi(\Delta t) / \psi(\Delta x)$. Note that, in the above matrix, $i=\sqrt{-1}$. Let $\eta=4 r^{2} \sin ^{2}(\beta \Delta x)$. We have the characteristic equation

$$
\lambda^{2}-(2-\eta) \lambda+1=0
$$

and the eigenvalues

$$
\lambda=1-\frac{1}{2} \eta \pm\left(\frac{1}{4} \eta^{2}-\eta\right)^{1 / 2}
$$

When $|\lambda| \leq 1$, that is, $r \leq 1$, the scheme (2.14) satisfies the Von Neumann conditions, which is a necessary condition of the stability of the scheme (2.14). If $r<1, \beta h \neq n \pi$, where $n$ is an integer, then $G$ has two different eigenvalues. If $r<1, \beta h=n \pi$, then $G$ is an identity matrix, but $\left(d / d_{\beta h}\right) G$ has two different eigenvalues [30]. So $r<1$ is the sufficient condition of the stability for integrator (2.14). Note that, if $r=1$, there is an unbounded solution $v_{i}^{j}=$ $(-1)^{i+j}(1-2 j), w_{i+1 / 2}^{j}=(-1)^{i+j} 2 j$. So the scheme (2.14) is not stable when $r=1$. Now, we find the necessary and sufficient condition of the stability for integrator (2.14), which is

$$
r=\frac{\phi(\Delta t)}{\psi(\Delta x)}<1
$$

With the consistence and stability conditions, we have following conclusion.

Theorem 2.2. The nonstandard finite difference variational integrator (2.14) is convergent, when the step sizes $\Delta t$ and $\Delta x$ satisfy $\phi(\Delta t)<\psi(\Delta x)$.

We have shown the idea of using the nonstandard finite difference method to get the discrete variational integrator and the corresponding discrete multisymplectic form formula. In the next section, we will consider the discrete variational integrators for a more complicated example, the nonlinear Klein-Gordon equation. 


\section{Nonstandard Finite Difference Variational Integrators for Nonlinear Klein-Gordon Equation}

In this section, we consider the nonlinear Klein-Gordon equation [31],

$$
u_{t t}=u_{x x}-u^{3}+u
$$

As known, this equation can be obtained by Euler-Lagrange equation (2.2) with the Lagrangian function

$$
L\left(u, u_{t}, u_{x}\right)=\frac{1}{2} u_{t}^{2}-\frac{1}{2} u_{x}^{2}-\frac{1}{4} u^{4}-\frac{1}{2} u^{2} .
$$

Now we consider the triangle discretization and square discretization, respectively, to get the nonstandard finite difference variational integrators.

\subsection{Triangle Discretization}

Following the steps in last section and using the idea of nonstandard finite difference, we define the discrete Lagrangian $L_{d}$ as

$$
\begin{aligned}
& L_{d}\left(u_{i}^{j}, u_{i+1}^{j}, u_{i}^{j+1}\right) \\
&=\frac{1}{2} \Delta t \Delta x\left(\frac{1}{2}\left(\frac{u_{i}^{j+1}-u_{i}^{j}}{\phi(\Delta t)}\right)^{2}-\frac{1}{2}\left(\frac{u_{i+1}^{j}-u_{i}^{j}}{\psi(\Delta x)}\right)^{2}\right. \\
&\left.-\frac{1}{4}\left(\frac{a_{1}\left(u_{i}^{j^{4}}+u_{i+1}^{j}{ }^{4}+u_{i}^{j+1^{4}}\right)+b_{1}\left(u_{i}^{j^{2}} u_{i+1}^{j} u_{i}^{j+1}+u_{i+1}^{j}{ }^{2} u_{i}^{j} u_{i}^{j+1}+u_{i}^{j+1^{2}} u_{i}^{j} u_{i+1}^{j}\right)}{3 a_{1}+3 b_{1}}\right)\right) \\
&\left.+\frac{1}{2}\left(\frac{a_{2}\left(u_{i}^{j^{2}}+u_{i+1}^{j}{ }^{2}+u_{i}^{j+1^{2}}\right)+b_{2}\left(u_{i}^{j} u_{i+1}^{j}+u_{i+1}^{j} u_{i}^{j+1}+u_{i}^{j} u_{i}^{j+1}\right)}{3 a_{2}+3 b_{2}}\right)\right),
\end{aligned}
$$

based on the following constructing rules,

(1) The discrete first derivative is represented by

$$
\frac{d u}{d t} \longrightarrow \frac{u_{i}^{j+1}-u_{i}^{j}}{\phi(\Delta t)}, \quad \frac{d u}{d x} \longrightarrow \frac{u_{i+1}^{j}-u_{i}^{j}}{\psi(\Delta x)}
$$


where the denominator functions are defined by (2.6), and

$$
\phi(\Delta t)=\Delta t+\mathcal{O}\left((\Delta t)^{3}\right), \quad \psi(\Delta x)=\Delta x+\mathcal{O}\left((\Delta x)^{3}\right) .
$$

(2) Nonlocal representations for $u^{4}$ and $u^{2}$ are given by

$$
\begin{gathered}
u^{4} \longrightarrow \frac{a_{1}\left(u_{i}^{j^{4}}+u_{i+1}^{j}{ }^{4}+u_{i}^{j+1^{4}}\right)+b_{1}\left(u_{i}^{j^{2}} u_{i+1}^{j} u_{i}^{j+1}+u_{i+1}^{j}{ }^{2} u_{i}^{j} u_{i}^{j+1}+u_{i}^{j+1^{2}} u_{i}^{j} u_{i+1}^{j}\right)}{3 a_{1}+3 b_{1}}, \\
u^{2} \longrightarrow \frac{a_{2}\left(u_{i}^{j^{2}}+u_{i+1}^{j}{ }^{2}+u_{i}^{j+1^{2}}\right)+b_{2}\left(u_{i}^{j} u_{i+1}^{j}+u_{i+1}^{j} u_{i}^{j+1}+u_{i}^{j} u_{i}^{j+1}\right)}{3 a_{2}+3 b_{2}},
\end{gathered}
$$

where $a_{1}, b_{1}, a_{2}$, and $b_{2}$ are positive parameters. Such discretizations for $u^{4}$ and $u^{2}$ guarantee the symmetric property of the discrete Lagrangian function [15].

Similarly, we define discrete Lagrangians on other two adjoint triangles,

$$
\begin{aligned}
& L_{d}\left(u_{i-1}^{j}, u_{i}^{j}, u_{i-1}^{j+1}\right) \\
&=\frac{1}{2} \Delta t \Delta x\left(\frac{1}{2}\left(\frac{u_{i-1}^{j+1}-u_{i-1}^{j}}{\phi(\Delta t)}\right)^{2}-\frac{1}{2}\left(\frac{u_{i}^{j}-u_{i-1}^{j}}{\psi(\Delta x)}\right)^{2}\right. \\
& \\
&-\frac{1}{4}\left(\frac{a_{1}\left(u_{i}^{j^{4}}+u_{i-1}^{j}{ }^{4}+u_{i-1}^{j+1^{4}}\right)+b_{1}\left({u_{i-1}^{j}}^{2} u_{i}^{j} u_{i-1}^{j+1}+u_{i}^{j^{2}} u_{i-1}^{j} u_{i-1}^{j+1}+u_{i-1}^{j+1^{2}} u_{i-1}^{j} u_{i}^{j}\right)}{3 a_{1}+3 b_{1}}\right) \\
&\left.+\frac{1}{2}\left(\frac{a_{2}\left(u_{i-1}^{j}{ }^{2}+u_{i}^{j^{2}}+u_{i-1}^{j+1^{2}}\right)+b_{2}\left(u_{i-1}^{j} u_{i}^{j}+u_{i}^{j} u_{i-1}^{j+1}+u_{i-1}^{j+1} u_{i-1}^{j}\right)}{3 a_{2}+3 b_{2}}\right)\right),
\end{aligned}
$$




$$
\begin{aligned}
& L_{d}\left(u_{i}^{j-1}, u_{i+1}^{j-1}, u_{i}^{j}\right) \\
& =\frac{1}{2} \Delta t \Delta x\left(\frac{1}{2}\left(\frac{u_{i}^{j}-u_{i}^{j-1}}{\phi(\Delta t)}\right)^{2}-\frac{1}{2}\left(\frac{u_{i+1}^{j-1}-u_{i}^{j-1}}{\psi(\Delta x)}\right)^{2}\right. \\
& -\frac{1}{4}\left(\frac{a_{1}\left(u_{i}^{j-1^{4}}+u_{i+1}^{j-1^{4}}+u_{i}^{j^{4}}\right)+b_{1}\left({u_{i}^{j-1}}^{2} u_{i+1}^{j-1} u_{i}^{j}+u_{i+1}^{j-1^{2}} u_{i}^{j} u_{i}^{j-1}+u_{i}^{j^{2}} u_{i}^{j-1} u_{i+1}^{j-1}\right)}{3 a_{1}+3 b_{1}}\right) \\
& \left.+\frac{1}{2}\left(\frac{a_{2}\left(u_{i}^{j-1^{2}}+u_{i+1}^{j-1^{2}}+u_{i}^{j^{2}}\right)+b_{2}\left(u_{i}^{j-1} u_{i+1}^{j-1}+u_{i+1}^{j-1} u_{i}^{j}+u_{i}^{j} u_{i}^{j-1}\right)}{3 a_{2}+3 b_{2}}\right)\right) .
\end{aligned}
$$

Now, the discrete variational integrator with nonstandard finite difference methods could be obtained by discrete Euler-Lagrange equation (2.12):

$$
D_{1} L_{d}\left(u_{i}^{j}, u_{i+1}^{j}, u_{i}^{j+1}\right)+D_{2} L_{d}\left(u_{i-1}^{j}, u_{i}^{j}, u_{i-1}^{j+1}\right)+D_{3} L_{d}\left(u_{i}^{j-1}, u_{i+1}^{j-1}, u_{i}^{j}\right)=0
$$

Substituting $L_{d}\left(u_{i}^{j}, u_{i+1}^{j}, u_{i}^{j+1}\right), L_{d}\left(u_{i-1}^{j}, u_{i}^{j}, u_{i-1}^{j+1}\right)$, and $L_{d}\left(u_{i}^{j-1}, u_{i+1}^{j-1}, u_{i}^{j}\right)$ into above equation, we arrive at

$$
\begin{aligned}
& -\frac{u_{i}^{j+1}-2 u_{i}^{j}+u_{i}^{j-1}}{(\phi(\Delta t))^{2}}+\frac{u_{i+1}^{j}-2 u_{i}^{j}+u_{i-1}^{j}}{(\psi(\Delta x))^{2}} \\
& -\frac{1}{4} \frac{1}{3 a_{1}+3 b_{1}}\left[12 a_{1} u_{i}^{j}+2 b_{1}\left(u_{i}^{j} u_{i+1}^{j} u_{i}^{j+1}+u_{i}^{j} u_{i-1}^{j} u_{i-1}^{j+1}+u_{i}^{j} u_{i}^{j-1} u_{i+1}^{j-1}\right)\right.
\end{aligned}
$$

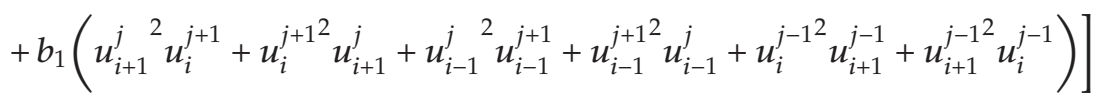

$$
\begin{aligned}
& +\frac{1}{2} \frac{1}{3 a_{2}+3 b_{2}}\left[6 a_{2} u_{i}^{j}+b_{2}\left(u_{i+1}^{j}+u_{i}^{j+1}+u_{i-1}^{j}+u_{i-1}^{j+1}+u_{i+1}^{j-1}+u_{i}^{j-1}\right)\right]=0 .
\end{aligned}
$$

Using the definition of discrete Lagrangian functions, one can find that this scheme is symmetric with respect to $(i, j+1)$ and $(i, j-1),(i+1, j)$ and $(i-1, j)$; that it is multisymplectic, and that it preserves the multisymplectic structure of the original equation. 
Its corresponding discrete multisymplectic form formula can be obtained from (2.17), that is,

$$
\begin{aligned}
& -\frac{d u_{i}^{j+1} \wedge d u_{i}^{j}-d u_{i}^{j} \wedge d u_{i}^{j-1}}{(\phi(\Delta t))^{2}}+\frac{d u_{i+1}^{j} \wedge d u_{i}^{j}-d u_{i}^{j} \wedge d u_{i-1}^{j}}{(\psi(\Delta x))^{2}} \\
& -\left(\left(C_{1} u_{i}^{j} u_{i}^{j+1}+C_{1} u_{i+1}^{j} u_{i}^{j+1}+\frac{1}{2} C_{1} u_{i}^{j+1^{2}}-C_{2}\right) d u_{i+1}^{j} \wedge d u_{i}^{j}\right. \\
& +\left(C_{1} u_{i}^{j} u_{i+1}^{j}+\frac{1}{2} C_{1} u_{i+1}^{j}{ }^{2}+C_{1} u_{i}^{j+1} u_{i+1}^{j}-C_{2}\right) d u_{i}^{j+1} \wedge d u_{i}^{j} \\
& +\left(C_{1} u_{i}^{j} u_{i-1}^{j+1}+C_{1} u_{i-1}^{j} u_{i-1}^{j+1}+\frac{1}{2} C_{1} u_{i-1}^{j+1^{2}}-C_{2}\right) d u_{i-1}^{j} \wedge d u_{i}^{j} \\
& +\left(C_{1} u_{i}^{j} u_{i-1}^{j}+\frac{1}{2} C_{1} u_{i-1}^{j}{ }^{2}+C_{1} u_{i-1}^{j+1} u_{i-1}^{j}-C_{2}\right) d u_{i-1}^{j+1} \wedge d u_{i}^{j} \\
& +\left(C_{1} u_{i}^{j} u_{i+1}^{j-1}+C_{1} u_{i}^{j-1} u_{i+1}^{j-1}+\frac{1}{2} C_{1} u_{i+1}^{j-1^{2}}-C_{2}\right) d u_{i}^{j-1} \wedge d u_{i}^{j} \\
& \left.+\left(C_{1} u_{i}^{j} u_{i}^{j-1}+\frac{1}{2} C_{1} u_{i}^{j-12}+C_{1} u_{i+1}^{j-1} u_{i}^{j-1}-C_{2}\right) d u_{i+1}^{j-1} \wedge d u_{i}^{j}\right)=0,
\end{aligned}
$$

where

$$
C_{1}=\frac{b_{1}}{2\left(3 a_{1}+3 b_{1}\right)}, \quad C_{2}=\frac{b_{2}}{3 a_{2}+3 b_{2}} .
$$

It shows the multisymplectic structure of scheme (3.9), and the relations between the field values on the three adjoint triangles are around $u_{i}^{j}$.

We now analyze the truncation error of integrator (3.9). By Taylor series expansion $[32,33]$, we have

$$
\begin{aligned}
& \frac{1}{4} \frac{1}{3 a_{1}+3 b_{1}}\left[12 a_{1} u_{i}^{j}+2 b_{1}\left(u_{i}^{j} u_{i+1}^{j} u_{i}^{j+1}+u_{i}^{j} u_{i-1}^{j} u_{i-1}^{j+1}+u_{i}^{j} u_{i}^{j-1} u_{i+1}^{j-1}\right)\right. \\
& \left.\quad+b_{1}\left(u_{i+1}^{j}{ }^{2} u_{i}^{j+1}+u_{i}^{j+1^{2}} u_{i+1}^{j}+u_{i-1}^{j}{ }^{2} u_{i-1}^{j+1}+u_{i-1}^{j+1^{2}} u_{i-1}^{j}+u_{i}^{j-1^{2}} u_{i+1}^{j-1}+u_{i+1}^{j-1} u_{i}^{j-1}\right)\right] \\
& =u^{3}\left(x_{i}, t_{j}\right)+\mathcal{O}\left((\Delta x)^{2}+\Delta x \Delta t+(\Delta t)^{2}\right), \\
& \frac{1}{2} \frac{1}{3 a_{2}+3 b_{2}}\left[6 a_{2} u_{i}^{j}+b_{2}\left(u_{i+1}^{j}+u_{i}^{j+1}+u_{i-1}^{j}+u_{i-1}^{j+1}+u_{i+1}^{j-1}+u_{i}^{j-1}\right)\right] \\
& =u\left(x_{i}, t_{j}\right)+\mathcal{O}\left((\Delta x)^{2}+(\Delta t)^{2}\right) .
\end{aligned}
$$


Combining the above two equations and (2.22), (2.23), we can observe that the nonstandard finite difference variational integrator (3.9) has the truncation error $\mathcal{O}\left((\Delta x)^{2}+\right.$ $\left.\Delta x \Delta t+(\Delta t)^{2}\right)$.

The above results are summarized in the following theorem.

Theorem 3.1. The nonstandard finite difference variational integrator (3.9) for the nonlinear KleinGordon equation (3.1) is multisymplectic, and its truncation error is $\mathcal{O}\left((\Delta x)^{2}+\Delta x \Delta t+(\Delta t)^{2}\right)$. The discrete multisymplectic structure of this scheme is presented by (3.10).

\subsection{Square Discretization}

In this case, we denote a square at $(i, j)$ with four ordered quaternion $((i, j),(i+1, j),(i+1, j+$ $1)$, and $(i, j+1))$ by $\square_{i}^{j}$ and define $X_{\square}$ to be the set of all such squares. Then the discrete jet bundle $[6,10]$ is defined as

$$
J_{\square}^{1} Y:=\left\{\left(u_{i}^{j}, u_{i+1}^{j}, u_{i+1}^{j+1}, u_{i}^{j+1}\right) \in \mathbb{R}^{4}:((i, j),(i+1, j),(i+1, j+1),(i, j+1)) \in X_{\square}\right\},
$$

which is equal to $X_{\square} \times \mathbb{R}^{4}$.

Following the philosophy of the nonstandard finite difference method, we define the discrete Lagrangian $L_{d}$ on $J_{\square}^{1} Y$ as

$$
\begin{aligned}
L_{d}\left(u_{i}^{j}, u_{i+1}^{j}, u_{i+1}^{j+1}, u_{i}^{j+1}\right) & \\
= & \left(\frac{1}{2}\left(\frac{u_{i}^{j+1}-u_{i}^{j}}{2 \phi(\Delta t)}+\frac{u_{i+1}^{j+1}-u_{i+1}^{j}}{2 \phi(\Delta t)}\right)^{2}-\frac{1}{2}\left(\frac{u_{i+1}^{j+1}-u_{i}^{j+1}}{2 \psi(\Delta x)}+\frac{u_{i+1}^{j}-u_{i}^{j}}{2 \psi(\Delta x)}\right)^{2}-\frac{1}{4} u_{i}^{j} u_{i+1}^{j} u_{i+1}^{j+1} u_{i}^{j+1}\right. \\
& \left.+\frac{1}{2}\left(\frac{u_{i}^{j} u_{i+1}^{j}+u_{i}^{j} u_{i+1}^{j+1}+u_{i}^{j} u_{i}^{j+1}+u_{i+1}^{j} u_{i+1}^{j+1}+u_{i+1}^{j+1} u_{i}^{j+1}+u_{i+1}^{j+1} u_{i}^{j+1}}{6}\right)\right) \Delta t \Delta x .
\end{aligned}
$$

In this case,

(1) the discrete first-derivative is represented by

$$
\begin{aligned}
& \frac{d u}{d t} \longrightarrow \frac{u_{i}^{j+1}-u_{i}^{j}}{2 \phi(\Delta t)}+\frac{u_{i+1}^{j+1}-u_{i+1}^{j}}{2 \phi(\Delta t)}, \\
& \frac{d u}{d x} \longrightarrow \frac{u_{i+1}^{j+1}-u_{i}^{j+1}}{2 \psi(\Delta x)}+\frac{u_{i+1}^{j}-u_{i}^{j}}{2 \psi(\Delta x)},
\end{aligned}
$$

where the denominator functions are defined by (2.6), and

$$
\phi(\Delta t)=\Delta t+\mathcal{O}\left((\Delta t)^{3}\right), \quad \psi(\Delta x)=\Delta x+\mathcal{O}\left((\Delta x)^{3}\right)
$$


(2) nonlocal representations for $u^{4}$ and $u^{2}$ are

$$
\begin{gathered}
u^{4} \longrightarrow u_{i}^{j} u_{i+1}^{j} u_{i+1}^{j+1} u_{i}^{j+1}, \\
u^{2} \longrightarrow \frac{u_{i}^{j} u_{i+1}^{j}+u_{i}^{j} u_{i+1}^{j+1}+u_{i}^{j} u_{i}^{j+1}+u_{i+1}^{j} u_{i+1}^{j+1}+u_{i+1}^{j+1} u_{i}^{j+1}+u_{i+1}^{j+1} u_{i}^{j+1}}{6}
\end{gathered}
$$

Similarly, we have the definitions of $L_{d}$ on the other three squares adjoint to $u_{i}^{j}$ :

$$
\begin{aligned}
& L_{d}\left(u_{i-1}^{j}, u_{i}^{j}, u_{i}^{j+1}, u_{i-1}^{j+1}\right) \\
& =\left(\frac{1}{2}\left(\frac{u_{i}^{j+1}-u_{i}^{j}}{2 \phi(\Delta t)}+\frac{u_{i-1}^{j+1}-u_{i-1}^{j}}{2 \phi(\Delta t)}\right)^{2}-\frac{1}{2}\left(\frac{u_{i}^{j}-u_{i-1}^{j}}{2 \psi(\Delta x)}+\frac{u_{i}^{j+1}-u_{i-1}^{j+1}}{2 \psi(\Delta x)}\right)^{2}\right. \\
& -\frac{1}{4} u_{i-1}^{j} u_{i}^{j} u_{i}^{j+1} u_{i-1}^{j+1} \\
& \left.+\frac{1}{2}\left(\frac{u_{i-1}^{j} u_{i}^{j}+u_{i-1}^{j} u_{i}^{j+1}+u_{i-1}^{j} u_{i-1}^{j+1}+u_{i}^{j} u_{i}^{j+1}+u_{i}^{j} u_{i-1}^{j+1}+u_{i}^{j+1} u_{i-1}^{j+1}}{6}\right)\right) \Delta t \Delta x, \\
& L_{d}\left(u_{i-1}^{j-1}, u_{i}^{j-1}, u_{i}^{j}, u_{i-1}^{j}\right) \\
& =\left(\frac{1}{2}\left(\frac{u_{i-1}^{j}-u_{i-1}^{j-1}}{2 \phi(\Delta t)}+\frac{u_{i}^{j}-u_{i}^{j-1}}{2 \phi(\Delta t)}\right)^{2}-\frac{1}{2}\left(\frac{u_{i}^{j}-u_{i-1}^{j}}{2 \psi(\Delta x)}+\frac{u_{i}^{j-1}-u_{i-1}^{j-1}}{2 \psi(\Delta x)}\right)^{2}\right. \\
& -\frac{1}{4} u_{i-1}^{j-1} u_{i}^{j-1} u_{i}^{j} u_{i-1}^{j} \\
& \left.+\frac{1}{2}\left(\frac{u_{i-1}^{j-1} u_{i}^{j-1}+u_{i-1}^{j-1} u_{i}^{j}+u_{i-1}^{j-1} u_{i-1}^{j}+u_{i}^{j-1} u_{i}^{j}+u_{i}^{j-1} u_{i-1}^{j}+u_{i}^{j} u_{i-1}^{j}}{6}\right)\right) \Delta t \Delta x, \\
& L_{d}\left(u_{i}^{j-1}, u_{i+1}^{j-1}, u_{i+1}^{j}, u_{i}^{j}\right) \\
& =\left(\frac{1}{2}\left(\frac{u_{i}^{j}-u_{i}^{j-1}}{2 \phi(\Delta t)}+\frac{u_{i+1}^{j}-u_{i+1}^{j-1}}{2 \phi(\Delta t)}\right)^{2}-\frac{1}{2}\left(\frac{u_{i+1}^{j}-u_{i}^{j}}{2 \psi(\Delta x)}+\frac{u_{i+1}^{j-1}-u_{i}^{j-1}}{2 \psi(\Delta x)}\right)^{2}\right. \\
& -\frac{1}{4} u_{i}^{j-1} u_{i+1}^{j-1} u_{i+1}^{j} u_{i}^{j} \\
& \left.+\frac{1}{2}\left(\frac{u_{i}^{j-1} u_{i+1}^{j-1}+u_{i}^{j-1} u_{i+1}^{j}+u_{i}^{j-1} u_{i}^{j}+u_{i+1}^{j-1} u_{i+1}^{j}+u_{i+1}^{j-1} u_{i}^{j}+u_{i+1}^{j} u_{i}^{j}}{6}\right)\right) \Delta t \Delta x .
\end{aligned}
$$


Journal of Applied Mathematics

Taking derivate of action functional with respect to $u_{i}^{j}$, we have the discrete EulerLagrange equation in this square discretization $[5,6,10,34]$, which is

$$
\begin{aligned}
& D_{1} L_{d}\left(u_{i}^{j}, u_{i+1}^{j}, u_{i+1}^{j+1}, u_{i}^{j+1}\right)+D_{2} L_{d}\left(u_{i-1}^{j}, u_{i}^{j}, u_{i}^{j+1}, u_{i-1}^{j+1}\right) \\
& \quad+D_{3} L_{d}\left(u_{i-1}^{j-1}, u_{i}^{j-1}, u_{i}^{j}, u_{i-1}^{j}\right)+D_{4} L_{d}\left(u_{i}^{j-1}, u_{i+1}^{j-1}, u_{i+1}^{j}, u_{i}^{j}\right)=0 .
\end{aligned}
$$

Substituting the discrete Lagrangian $L_{d}\left(u_{i}^{j}, u_{i+1}^{j}, u_{i+1}^{j+1}, u_{i}^{j+1}\right), L_{d}\left(u_{i-1}^{j}, u_{i}^{j}, u_{i}^{j+1}, u_{i-1}^{j+1}\right)$, $L_{d}\left(u_{i-1}^{j-1}, u_{i}^{j-1}, u_{i}^{j}, u_{i-1}^{j}\right)$, and $L_{d}\left(u_{i}^{j-1}, u_{i+1}^{j-1}, u_{i+1}^{j}, u_{i}^{j}\right)$ into the previous equation, we arrive at

$$
\begin{aligned}
& \left(-\frac{1}{2 \phi(\Delta t)^{2}}-\frac{1}{2 \psi(\Delta x)^{2}}+\frac{1}{6}\right)\left(u_{i}^{j+1}+u_{i}^{j-1}\right)+\left(\frac{1}{2 \phi(\Delta t)^{2}}+\frac{1}{2 \psi(\Delta x)^{2}}+\frac{1}{6}\right)\left(u_{i+1}^{j}+u_{i-1}^{j}\right) \\
& \quad+\left(\frac{1}{\phi(\Delta t)^{2}}-\frac{1}{\psi(\Delta x)^{2}}\right) u_{i}^{j}+\left(-\frac{1}{4 \phi(\Delta t)^{2}}+\frac{1}{4 \psi(\Delta x)^{2}}+\frac{1}{12}\right)\left(u_{i+1}^{j+1}+u_{i-1}^{j+1}+u_{i-1}^{j-1}+u_{i+1}^{j-1}\right) \\
& \quad-\frac{1}{4}\left(u_{i+1}^{j} u_{i+1}^{j+1} u_{i}^{j+1}+u_{i-1}^{j} u_{i}^{j+1} u_{i-1}^{j+1}+u_{i-1}^{j-1} u_{i}^{j-1} u_{i-1}^{j}+u_{i}^{j-1} u_{i+1}^{j-1} u_{i+1}^{j}\right)=0 .
\end{aligned}
$$

After simple calculations, it becomes

$$
\begin{aligned}
\frac{1}{4}\left(\frac{u_{i+1}^{j+1}-2 u_{i+1}^{j}+u_{i+1}^{j-1}}{(\phi(\Delta t))^{2}}+2 \frac{u_{i}^{j+1}-2 u_{i}^{j}+u_{i}^{j-1}}{(\phi(\Delta t))^{2}}+\frac{u_{i-1}^{j+1}-2 u_{i-1}^{j}+u_{i-1}^{j-1}}{(\phi(\Delta t))^{2}}\right) \\
\quad-\frac{1}{4}\left(\frac{u_{i+1}^{j+1}-2 u_{i}^{j+1}+u_{i-1}^{j+1}}{(\psi(\Delta x))^{2}}+2 \frac{u_{i+1}^{j}-2 u_{i}^{j}+u_{i-1}^{j}}{(\psi(\Delta x))^{2}}+\frac{u_{i+1}^{j-1}-2 u_{i}^{j-1}+u_{i-1}^{j-1}}{(\psi(\Delta x))^{2}}\right) \\
\quad+\frac{1}{4}\left(u_{i+1}^{j} u_{i+1}^{j+1} u_{i}^{j+1}+u_{i-1}^{j} u_{i}^{j+1} u_{i-1}^{j+1}+u_{i-1}^{j-1} u_{i}^{j-1} u_{i-1}^{j}+u_{i}^{j-1} u_{i+1}^{j-1} u_{i+1}^{j}\right) \\
\quad-\frac{1}{12}\left(u_{i+1}^{j+1}+u_{i-1}^{j+1}+u_{i-1}^{j-1}+u_{i+1}^{j-1}+2 u_{i}^{j+1}+2 u_{i}^{j-1}+2 u_{i+1}^{j}+2 u_{i-1}^{j}\right)=0 .
\end{aligned}
$$

It is multisymplectic and symmetric in $(i, j+1)$ and $(i, j-1),(i+1, j)$ and $(i-1, j)$. Similarly, we have the discrete multisymplectic form formula:

$$
\begin{aligned}
& \left(-\frac{1}{2(\phi(\Delta t))^{2}}-\frac{1}{2(\psi(\Delta x))^{2}}+\frac{1}{6}-\frac{1}{4} u_{i+1}^{j} u_{i+1}^{j+1}-\frac{1}{4} u_{i-1}^{j} u_{i-1}^{j+1}\right) d u_{i}^{j+1} \wedge d u_{i}^{j} \\
& \quad+\left(-\frac{1}{2(\phi(\Delta t))^{2}}-\frac{1}{2(\psi(\Delta x))^{2}}+\frac{1}{6}-\frac{1}{4} u_{i-1}^{j-1} u_{i}^{j+1}-\frac{1}{4} u_{i+1}^{j-1} u_{i+1}^{j}\right) d u_{i}^{j-1} \wedge d u_{i}^{j} \\
& \quad+\left(\frac{1}{2(\phi(\Delta t))^{2}}+\frac{1}{2(\psi(\Delta x))^{2}}+\frac{1}{6}-\frac{1}{4} u_{i+1}^{j+1} u_{i}^{j+1}-\frac{1}{4} u_{i}^{j-1} u_{i+1}^{j-1}\right) d u_{i+1}^{j} \wedge d u_{i}^{j}
\end{aligned}
$$




$$
\begin{aligned}
& +\left(\frac{1}{2(\phi(\Delta t))^{2}}+\frac{1}{2(\psi(\Delta x))^{2}}+\frac{1}{6}-\frac{1}{4} u_{i}^{j+1} u_{i-1}^{j+1}-\frac{1}{4} u_{i-1}^{j-1} u_{i}^{j-1}\right) d u_{i}^{j-1} \wedge d u_{i}^{j} \\
& +\left(-\frac{1}{4(\phi(\Delta t))^{2}}+\frac{1}{4(\psi(\Delta x))^{2}}+\frac{1}{12}-\frac{1}{4} u_{i+1}^{j} u_{i}^{j+1}\right) d u_{i+1}^{j+1} \wedge d u_{i}^{j} \\
& +\left(-\frac{1}{4(\phi(\Delta t))^{2}}+\frac{1}{4(\psi(\Delta x))^{2}}+\frac{1}{12}-\frac{1}{4} u_{i-1}^{j} u_{i}^{j+1}\right) d u_{i-1}^{j+1} \wedge d u_{i}^{j} \\
& +\left(-\frac{1}{4(\phi(\Delta t))^{2}}+\frac{1}{4(\psi(\Delta x))^{2}}+\frac{1}{12}-\frac{1}{4} u_{i}^{j-1} u_{i-1}^{j}\right) d u_{i-1}^{j-1} \wedge d u_{i}^{j} \\
& +\left(-\frac{1}{4(\phi(\Delta t))^{2}}+\frac{1}{4(\psi(\Delta x))^{2}}+\frac{1}{12}-\frac{1}{4} u_{i}^{j-1} u_{i+1}^{j+1}\right) d u_{i+1}^{j-1} \wedge d u_{i}^{j}=0 .
\end{aligned}
$$

To study the truncation error of the integrator, we do the Taylor expansion which leads to

$$
\begin{gathered}
\frac{u_{p}^{j+1}-2 u_{p}^{j}+u_{p}^{j-1}}{(\phi(\Delta t))^{2}}=u_{t t}\left(x_{p}, t_{j}\right)+\mathcal{O}\left((\Delta t)^{2}\right), \quad p=i-1, i, i+1, \\
\frac{u_{i+1}^{q}-2 u_{i}^{q}+u_{i-1}^{q}}{(\psi(\Delta x))^{2}}=u_{x x}\left(x_{i}, t_{q}\right)+\mathcal{O}\left((\Delta x)^{2}\right), \quad q=j-1, j, j+1, \\
\frac{1}{4}\left(u_{i+1}^{j} u_{i+1}^{j+1} u_{i}^{j+1}+u_{i-1}^{j} u_{i}^{j+1} u_{i-1}^{j+1}+u_{i-1}^{j-1} u_{i}^{j-1} u_{i-1}^{j}+u_{i}^{j-1} u_{i+1}^{j-1} u_{i+1}^{j}\right) \\
=u^{3}\left(x_{i}, t_{j}\right)+(\Delta x)^{2}\left(2 u\left(x_{i}, t_{j}\right) u_{x x}\left(x_{i}, t_{j}\right)+u_{x}^{2}\left(x_{i}, t_{j}\right)\right) \\
\quad+(\Delta t)^{2}\left(2 u\left(x_{i}, t_{j}\right) u_{t t}\left(x_{i}, t_{j}\right)+u_{t}^{2}\left(x_{i}, t_{j}\right)\right) \\
=u^{3}\left(x_{i}, t_{j}\right)+\mathcal{O}\left((\Delta x)^{2}+(\Delta t)^{2}\right), \\
\frac{1}{12}\left(u_{i+1}^{j+1}+u_{i-1}^{j+1}+u_{i-1}^{j-1}+u_{i+1}^{j-1}+2 u_{i}^{j+1}+2 u_{i}^{j-1}+2 u_{i+1}^{j}+2 u_{i-1}^{j}\right) \\
=u\left(x_{i}, t_{j}\right)+\mathcal{O}\left((\Delta x)^{2}+(\Delta t)^{2}\right) .
\end{gathered}
$$

Combing these equations, we can readily observe that the nonstandard finite difference variational integrator (3.21) has truncation error $\mathcal{O}\left((\Delta x)^{2}+(\Delta t)^{2}\right)$. To verify this conclusion, we investigate the numerical convergence order in our numerical experiments. See Section 4.

We summarize our conclusion in the following theorem. 


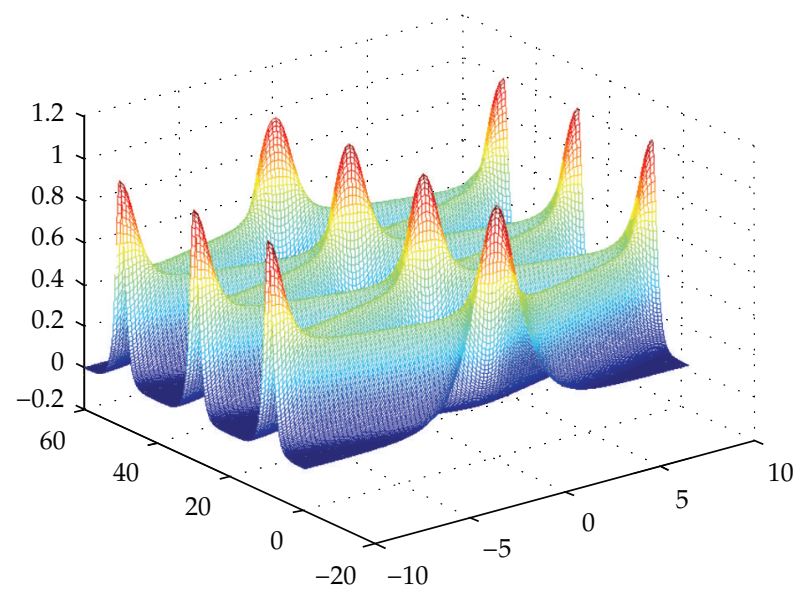

Figure 1: The waveforms of linear wave equation (2.1) by the nonstandard finite difference variational integrator (2.14) $(\Delta t=0.1, \Delta x=0.2)$.

Theorem 3.2. The nonstandard finite difference variational integrator (3.21) for the nonlinear KleinGordon equation (3.1) is multisymplectic, and its truncation error is $\mathcal{O}\left((\Delta x)^{2}+(\Delta t)^{2}\right)$. The discrete multisymplectic structure of this scheme is presented by (3.22).

\section{Numerical Simulations}

In this section, we report the performance of the nonstandard finite difference variational integrator (2.14) for solving linear wave equation (2.1) and the nonstandard finite difference variational integrators (3.21) and (3.9) for the nonlinear Klein-Gordon equation (3.1).

\subsection{Linear Wave Equation}

For linear wave equation (2.1), we consider the initial conditions

$$
\begin{gathered}
u(x, 0)=\operatorname{sech} x, \quad-10<x<10, \\
u_{t}(x, 0)=0, \quad-10<x<10,
\end{gathered}
$$

and the periodic boundary conditions

$$
u(-10, t)=u(10, t), \quad u_{x}(-10, t)=u_{x}(10, t)
$$

The nonstandard finite difference variational integrator (2.14) is an explicit five points scheme. We choose the denominator functions $\phi$ and $\psi$ in as $\phi(\Delta t)=2 \sin (\Delta t / 2)$ and $\psi(\Delta t)=$ $2 \sin (\Delta x / 2)$.

From Figure 1, we can see that the nonstandard finite difference variational integrator (2.14) for the linear wave equation performs very well and the periodicity of the linear wave equation is preserved accurately. 


\subsection{Nonlinear Klein-Gordon Equation}

We now consider the nonlinear Klein-Gordon equation (3.1) with the initial condition

$$
u(x, 0)=A\left(1+\cos \left(\frac{2 \pi x}{L}\right)\right), \quad u_{t}(x, 0)=0,
$$

and periodic boundary conditions.

We use nonstandard finite difference variational integrator (3.21) to simulate this problem with amplitude $A=5$. The nonstandard finite difference variational integrator (2.14) is an implicit nine-points nonstandard finite difference scheme. The denominator functions $\phi$ and $\psi$ are defined the same as before.

As depicted in Figure 2, the nonstandard finite difference variational integrator (3.21) simulates the wave propagation perfectly at the beginning. After a long time simulation, the integrator still performs very accurate and stable, without showing any blowup. With periodic boundary condition, the wave going out the computational domain shows up in the other direction periodically.

\subsection{Convergence Order of the Nonlinear Integrators (3.9) and (3.21)}

To further investigate the numerical convergence of the proposed schemes, we conduct a series of numerical tests of our nonlinear integrators. In this example, we consider the nonlinear Klein-Gordon equation (3.1) with the initial boundary conditions as follows:

$$
\begin{gathered}
u(x, 0)=\sqrt{2} \operatorname{sech}\left(\sqrt{\frac{-1}{1-c^{2}} x}\right), \\
u_{t}(x, 0)=c \sqrt{\frac{-2}{1-c^{2}}} \operatorname{sech}\left(\sqrt{\frac{-1}{1-c^{2}}}(x-c t)\right) \tanh \left(\sqrt{\frac{-1}{1-c^{2}}}(x-c t)\right), \\
u(-10, t)=\sqrt{2} \operatorname{sech}\left(\sqrt{\frac{-1}{1-c^{2}}}(-10-c t)\right), \\
u(10, t)=\sqrt{2} \operatorname{sech}\left(\sqrt{\frac{-1}{1-c^{2}}}(10-c t)\right)
\end{gathered}
$$

where $c=1.2$. The exact solution of the problem is

$$
u(x, t)=\sqrt{2} \operatorname{sech}\left(\sqrt{\frac{-1}{1-c^{2}}}(10-c t)\right) .
$$

The nonstandard finite difference integrators (3.9) and (3.21) are applied to simulate the Klein-Gordon equation. In the integrator (3.9), we choose $a_{1}=b_{1}=a_{2}=b_{2}=1$ here. 


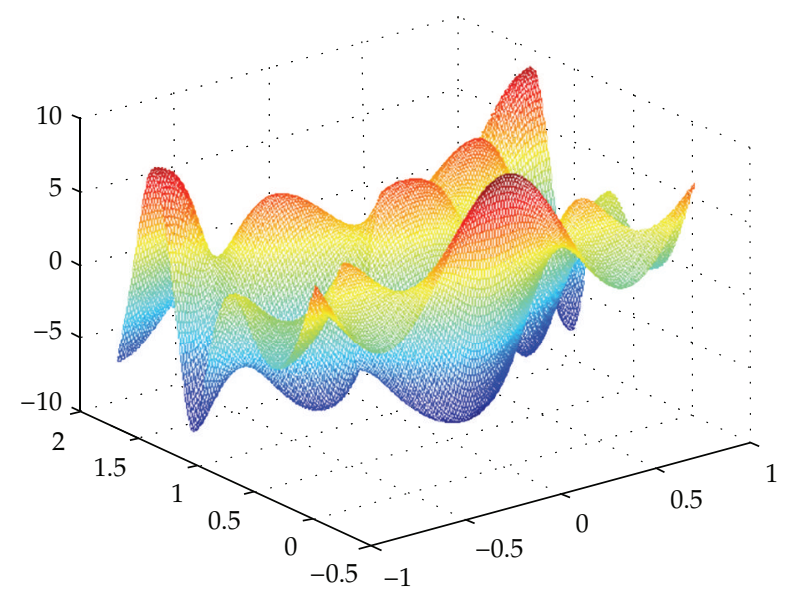

(a) Waveforms at the beginning from $t=0$ to $t=2$

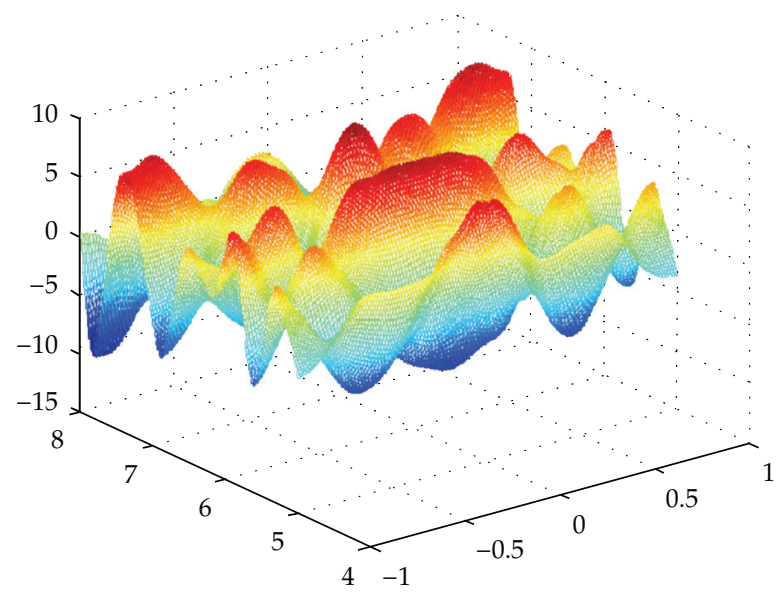

(b) Waveforms from $t=5$ to $t=8$

Figure 2: Waveforms of nonlinear Klein-Gordon equation by the nonstandard finite difference variational integrator (3.21) $(\Delta t=0.01, \Delta x=0.01)$.

The $l^{\infty}$-norm errors at $t=1, t=1.5$, and $t=2$ are listed in Tables 1 and 2 . The orders in the tables are calculated with the formula $[35,36]$

$$
\text { Order } \approx \frac{\ln \left(\operatorname{Error}\left(\Delta x_{1}\right) / \operatorname{Error}\left(\Delta x_{2}\right)\right)}{\ln \left(\Delta x_{1} / \Delta x_{2}\right)}
$$

Overall, it is clear that the error decreases as the mesh size goes to zero, indicating the convergence of our nonlinear integrators (3.9) and (3.21). Moreover, numerical orders clearly exhibit second order convergence when the mesh size decreases with $\Delta t=\Delta x$, which further confirms our theoretical derivation of the truncation errors of the numerical schemes.

In all, the numerical tests verify that the nonstandard finite difference variational integrators that we developed are capable of preserving the characteristics of original equations. They are all accurate, effective, and suitable for solving multisymplectic systems. 
Table 1: The error and convergence orders of (3.9) for Klein-Gordon problem with $\Delta t=\Delta x$.

\begin{tabular}{lccc}
\hline Mesh size & $t=1$ & $t=1.5$ & $t=2$ \\
\hline$\Delta x=0.1$ & $6.3 e-3$ & $8.9 e-3$ & $1.07 e-2$ \\
Error & - & - & - \\
Order & $1.6 e-3$ & $2.3 e-3$ & $2.8 e-3$ \\
\hline$\Delta x=0.05$ & 1.9773 & 1.9522 & 1.9341 \\
Error & & & $7.0059 e-4$ \\
Order & $4.1645 e-4$ & $5.8258 e-4$ & 1.9988 \\
\hline$\Delta x=0.025$ & 1.9419 & 1.9811 & $1.7565 e-4$ \\
Error & & & 1.9959 \\
Order & $1.0480 e-4$ & $1.4622 e-4$ & 1.9943 \\
\hline$\Delta x=0.0125$ & 1.9905 & & \\
Error & & & \\
Order & & & \\
\hline
\end{tabular}

Table 2: The error and convergence orders of (3.21) for Klein-Gordon problem with $\Delta t=\Delta x$.

\begin{tabular}{lccc}
\hline Mesh size & $t=1$ & $t=1.5$ & $t=2$ \\
\hline$\Delta x=0.1$ & $1.28 e-2$ & $1.81 e-2$ & $2.25 e-2$ \\
Error & - & - & - \\
Order & & & $5.7 e-3$ \\
\hline$\Delta x=0.05$ & $3.3 e-3$ & $4.6 e-3$ & 1.9809 \\
Error & 1.9556 & 1.9763 & $1.4 e-3$ \\
Order & & & 2.0255 \\
\hline$\Delta x=0.025$ & $8.3445 e-4$ & $1.2 e-3$ & $3.6187 e-4$ \\
Error & 1.9836 & 1.9386 & 1.9984 \\
Order & & & $2.9458 e-4$ \\
\hline$\Delta x=0.0125$ & $2.0990 e-4$ & 2.0263 & \\
Error & 1.9911 & & \\
Order & &
\end{tabular}

\section{Conclusion}

In this paper, we have considered a linear wave equation and a nonlinear Kleingordon equation. We have derived the nonstandard finite difference variational integrators and the corresponding multisymplectic form formulas from these two multisymplectic PDEs. We have shown that the nonstandard finite difference methods are flexible in constructing numerical schemes and can be employed to derive multisymiplectic schemes for multisymplectic systems. The convergence of our methods has been discussed. The numerical experiments have shown effectiveness and efficiency of these nonstandard finite difference variational integrators.

\section{Acknowledgments}

The authors are grateful to the editor and anonymous reviewers for their careful reading and many constructive suggestions which lead to a great improvement of this paper. This work 
is supported by the NNSF of China (no. 11271101) and the NNSF of Shandong Province (no. ZR2010AQ021).

\section{References}

[1] R. Abraham and J. E. Marsden, Foundations of Mechanics, Benjamin/Cummings Publishing, Reading, Mass, USA, 2nd edition, 1978.

[2] J.-B. Chen, "Variational integrators and the finite element method," Applied Mathematics and Computation, vol. 196, no. 2, pp. 941-958, 2008.

[3] J.-B. Chen, M.-Z. Qin, and R. Scherer, "Multisymplectic and variational integrators," International Journal of Pure and Applied Mathematics, vol. 44, no. 4, pp. 509-536, 2008.

[4] P. G. Ciarlet, A. Iserles, R. V. Kohn, and M. H. Wright, Simulating Hamltonian Dynamics, Cambridge Monographs on Applied and Computational Mathematics, Cambridge University Press, New York, NY, USA, 2004.

[5] J. E. Marsden and M. West, "Discrete mechanics and variational integrators," Acta Numerica, vol. 10, pp. 357-514, 2001.

[6] J. E. Marsden, G. W. Patrick, and S. Shkoller, "Multisymplectic geometry, variational integrators, and nonlinear PDEs," Communications in Mathematical Physics, vol. 199, no. 2, pp. 351-395, 1998.

[7] M. Leok and J. Zhang, "Discrete Hamiltonian variational integrators," IMA Journal of Numerical Analysis, vol. 31, no. 4, pp. 1497-1532, 2011.

[8] K. I. Nawafleh, "Canonical quantization of higher-order Lagrangians," Journal of Applied Mathematics, vol. 2011, Article ID 375838, 11 pages, 2011.

[9] S. Pekarsky and J. E. Marsden, "Abstract mechanical connection and abelian reconstruction for almost Kähler manifolds," Journal of Applied Mathematics, vol. 1, no. 1, pp. 1-28, 2001.

[10] J. Vankerschaver, C. Liao, and M. Leok, "Generating functionals and Lagrangian PDEs," Journal of Nonlinear Science. In press.

[11] R. E. Mickens, Applications of Nonstandard Finite Difference Schemes, World Scientific Publishing, Singapore, 2000.

[12] R. E. Mickens, "Nonstandard finite difference schemes for differential equations," Journal of Difference Equations and Applications, vol. 8, no. 9, pp. 823-847, 2002.

[13] R. E. Mickens, "A nonstandard finite difference scheme for the diffusionless Burgers equation with logistic reaction," Mathematics and Computers in Simulation, vol. 62, no. 1-2, pp. 117-124, 2003.

[14] R. E. Mickens, "Dynamic consistency: a fundamental principle for constructing nonstandard finite difference schemes for differential equations," Journal of Difference Equations and Applications, vol. 11, no. 7 , pp. 645-653, 2005.

[15] R. E. Mickens, "A numerical integration technique for conservative oscillators combining nonstandard finite-difference methods with a Hamilton's principle," Journal of Sound and Vibration, vol. 285, no. 1-2, pp. 477-482, 2005.

[16] A. J. Arenas, G. González-Parra, and B. M. Chen-Charpentier, "A nonstandard numerical scheme of predictor-corrector type for epidemic models," Computers \& Mathematics with Applications, vol. 59, no. 12, pp. 3740-3749, 2010.

[17] G. González-Parra, A. J. Arenas, and B. M. Chen-Charpentier, "Combination of nonstandard schemes and Richardson's extrapolation to improve the numerical solution of population models," Mathematical and Computer Modelling, vol. 52, no. 7-8, pp. 1030-1036, 2010.

[18] R. J. Villanueva, A. J. Arenas, and G. Gonzlez-Parra, "A nonstandard dynamically consistent numerical scheme applied to obesity dynamics," Journal of Applied Mathematics, vol. 2008, Article ID 640154, 14 pages, 2008.

[19] P. M. Jordan, "A nonstandard finite difference scheme for nonlinear heat transfer in a thin finite rod," Journal of Difference Equations and Applications, vol. 9, no. 11, pp. 1015-1021, 2003.

[20] A. Malek, "Applications of nonstandard finite difference methods to nonlinear heat transfer problems heat transfer-mathematical modelling," Numerical Methods and Information Technology. In press.

[21] Q. Ma, D. Ding, and X. Ding, "A nonstandard fnite-difference method for a linear oscillator with additive noise," Applied Mathematics and Information Science. In press.

[22] P. M. Manning and G. F. Margrave, "Introduction to non-standard fnite-difference modelling," CREWES Research Report 18, 2006.

[23] T. J. Bridges, "Multi-symplectic structures and wave propagation," Mathematical Proceedings of the Cambridge Philosophical Society, vol. 121, no. 1, pp. 147-190, 1997. 
[24] T. J. Bridges and S. Reich, "Numerical methods for Hamiltonian PDEs," Journal of Physics A, vol. 39, no. 19 , pp. $5287-5320,2006$.

[25] T. J. Bridges and S. Reich, "Multi-symplectic integrators: numerical schemes for Hamiltonian PDEs that conserve symplecticity," Physics Letters A, vol. 284, no. 4-5, pp. 184-193, 2001.

[26] J. Hong and Y. Liu, "A novel numerical approach to simulating nonlinear Schrödinger equations with varying coefficients," Applied Mathematics Letters, vol. 16, no. 5, pp. 759-765, 2003.

[27] J. Hong, Y. Liu, H. Munthe-Kaas, and A. Zanna, "Globally conservative properties and error estimation of a multi-symplectic scheme for Schrödinger equations with variable coefficients," Applied Numerical Mathematics, vol. 56, no. 6, pp. 814-843, 2006.

[28] X.-C. Li and W.-A. Yao, "Symplectic analytical solutions for the magnetoelectroelastic solids plane problem in rectangular domain," Journal of Applied Mathematics, vol. 2011, Article ID 165160, 15 pages, 2011.

[29] J. Vankerschaver and M. Leok, "A variational, second-order integrator for point vortices on the sphere," In press.

[30] T. Meis and U. Marcowitz, Numerical Solution of Partial Differential Equations, Springer, Berlin, Germany, 1981.

[31] A. M. A. El-Sayed, A. Elsaid, and D. Hammad, "A reliable treatment of homotopy perturbation method for solving the nonlinear klein-gordon equation of arbitrary (fractional) orders," Journal of Applied Mathematics, vol. 2012, Article ID 581481, 13 pages, 2012.

[32] J.-B. Chen, "A multisymplectic integrator for the periodic nonlinear Schrödinger equation," Applied Mathematics and Computation, vol. 170, no. 2, pp. 1394-1417, 2005.

[33] J.-B. Chen, M.-Z. Qin, and Y.-F. Tang, "Symplectic and multi-symplectic methods for the nonlinear Schrödinger equation," Computers \& Mathematics with Applications, vol. 43, no. 8-9, pp. 1095-1106, 2002.

[34] M. Leok and T. Shingel, "General techniques for constructing variational integrators," Frontiers of Mathematics in China, vol. 7, no. 2, pp. 273-303, 2012.

[35] S. Zhou and X. Cheng, "Numerical solution to coupled nonlinear Schrödinger equations on unbounded domains," Mathematics and Computers in Simulation, vol. 80, no. 12, pp. 2362-2373, 2010.

[36] S. Zhou and X. Cheng, "A linearly semi-implicit compact scheme for the Burgers-Huxley equation," International Journal of Computer Mathematics, vol. 88, no. 4, pp. 795-804, 2011. 


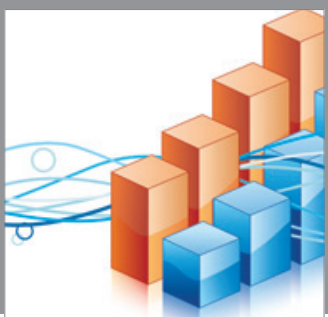

Advances in

Operations Research

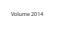

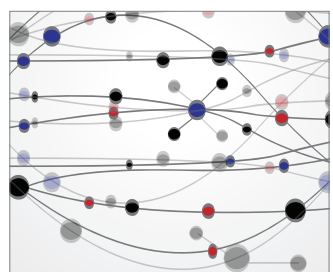

\section{The Scientific} World Journal
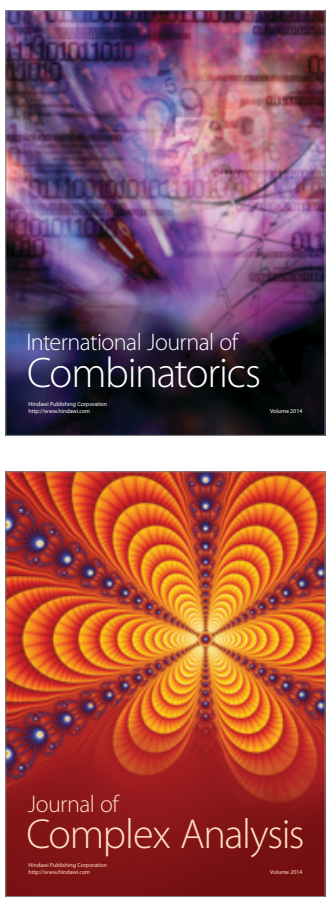

International Journal of

Mathematics and

Mathematical

Sciences
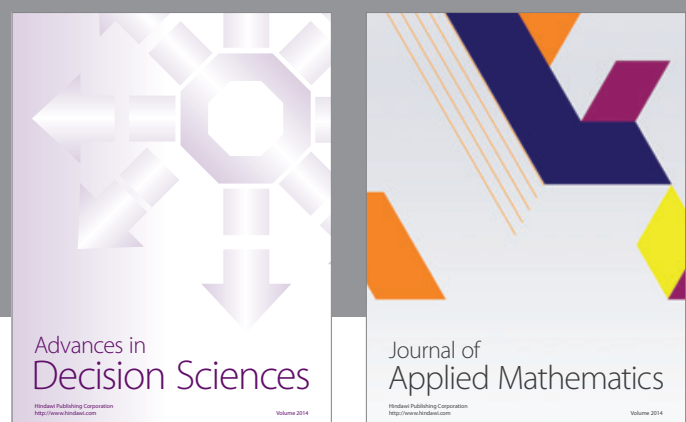

Journal of

Applied Mathematics
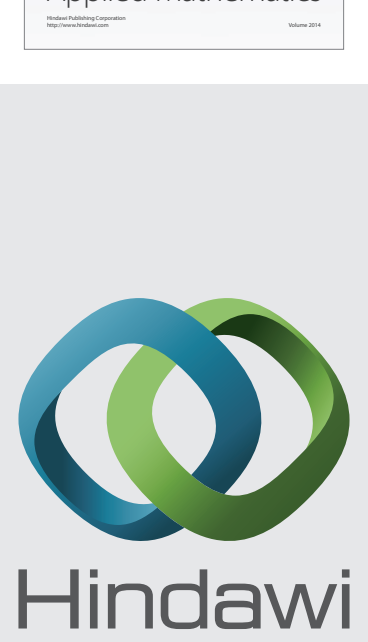

Submit your manuscripts at http://www.hindawi.com
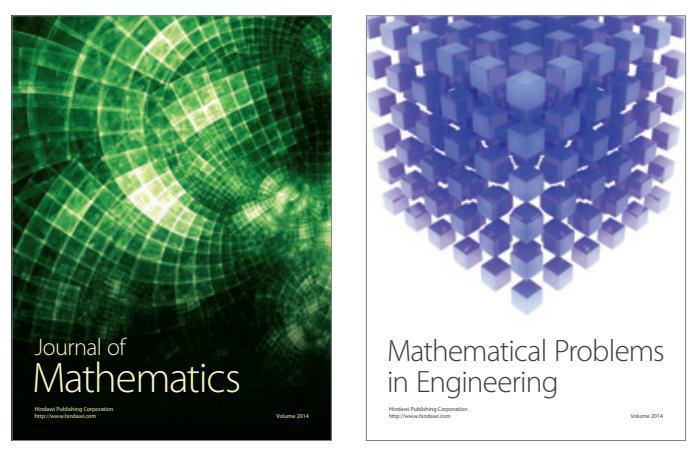

Mathematical Problems in Engineering
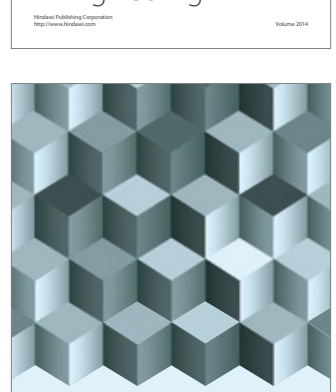

Journal of

Function Spaces
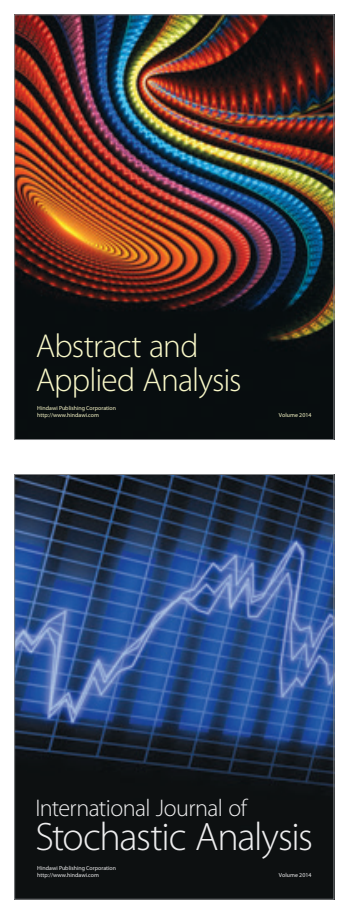

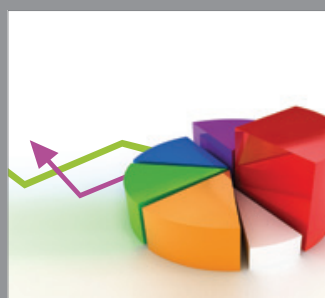

ournal of

Probability and Statistics

Promensencen
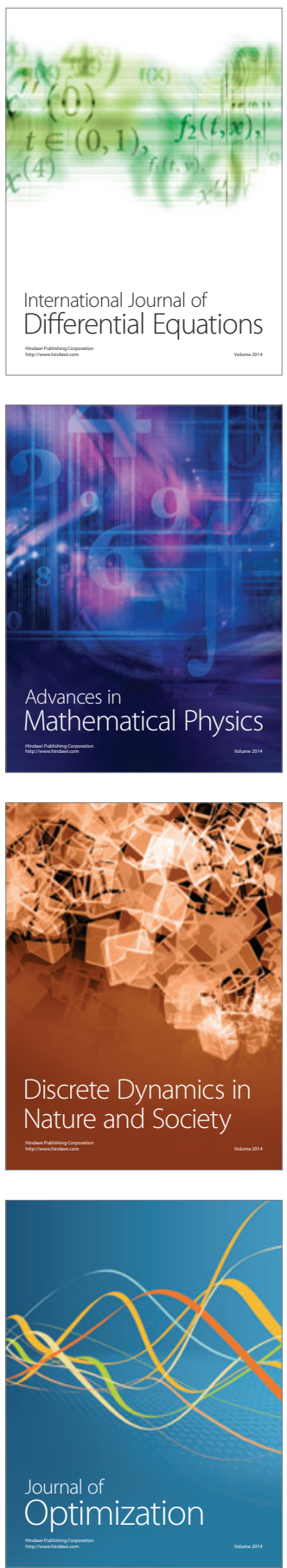